\title{
FAXBACK RESPONSE
}

Name:

\section{Address:}

\section{E-mail:}

\section{Specialty:}

FAX: 212.328.0600

MAIL:

CNS SPECTRUMS

\author{
MedWorks Media
}

333 Hudson Street, 7th Floor

New York, NY 10013

\section{Signature:}

\section{Date:}

Your comments are important to us. This easy-to-use form provides you with the opportunity to express your opinions. Our goal is to make CNS Spectrums your source for practical and clinical neuropsychiatric information. By filling out this FaxBack form, you will enable us to incorporate your views about our editorial content in future issues. Please fill out this form in its entirety. Thank you.

\section{On a scale of 1 to 5 ( $1=$ Poor, $5=$ Excellent), please indicate your level of interest and/or satisfaction with the editorial content in this issue.}

\section{Cover Story: Body Dysmorphic Disorder}

$\begin{array}{lllll}1 & 2 & 3 & 4 & 5\end{array}$

\section{Departments}

CNS News

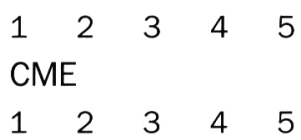

2. Which areas of neuropsychiatry would you like us to cover in the future?
3. Please describe your reading pattern for this issue:

O cover to cover

skim Table of Contents

select items of interest

O skim text

$O$ did not read

4. On a scale of 1 to 5 ( $1=$ Incomplete, $5=$ Comprehensive), how would you describe the depth of coverage for this issue?

$$
\begin{array}{lllll}
1 & 2 & 3 & 4 & 5
\end{array}
$$

5. Any other comments?

6. Please indicate your title:

psychiatrist

neurologist

When you send us this form, you'll receive one complimentary slide kit and one complimentary reference material (if available). Please make your selection below.

\section{SLIDE LIBRARY}

$\square$ Diagnosing and Treating Generalized Anxiety Disorder

$\square$ Managing Psychiatric Illness in the Elderly

$\square$ Diagnosis and Treatment of Anxiety Disorders in Children

$\square$ Optimal Uses of Antidepressants

$\square$ Immunogenicity of Botulinum Toxin Therapy

$\square$ Recent Advances in the Treatment of GAD

$\square$ Cancer Treatment: Angiogenesis $\mathcal{E}$ Signal Transduction

$\square$ Comorbidity and Quality of Life Issues in GAD
$\square$ Current Treatments of ADHD
$\square$ New Developments in the Treatment of Epilepsy
$\square$ Current and Emerging Treatments for Cervical Dystonia
$\square$ Remission-Oriented Treatment of Depression
$\square$ Remission-Oriented Treatment of GAD
$\square$ Atypical Antipsychotics and Diabetes Mellitus
$\square$ Recent Phase III Studies: Colorectal Cancer 


\section{Introduction}

CNS Spectrums is an Index Medicus journal that publishes original scientific literature and reviews on a wide variety of neuroscientific topics of interest to the clinician. CNS Spectrums will publish 12 issues in 2002. As the immense prevalence of comorbid diseases among patients seen by psychiatrists and neurologists increases, these physicians will jointly diagnose and treat the neuropsychiatrically ill. Our mission is to provide these physicians with an editorial package that will enhance and increase their understanding of neuropsychiatry; therefore, manuscripts that address crossover issues germane to neurology and psychiatry will be given immediate priority.

\section{Scope of Manuscripts}

CNS Spectrums will consider the following types of articles for publication:

Original Reports: Original reports present methodologically sound original data.

Reviews: Reviews are overview articles that summarize and synthesize the literature on various topics in a scholarly and clinically relevant fashion. Suitable topics include mood disorders, schizophrenia and related disorders, personality disorders, substanceuse disorders, anxiety disorders, neuroscience, psychosocial aspects of psychiatry, child psychiatry, geriatric psychiatry, and other topics of interest to clinicians. Original flowcharts designed to aid the clinician in diagnosis and treatment will be considered for publication in reviews and are encouraged.

Case Reports: Single or multiple case reports will be considered for publication.

Letters to the Editor: Letters will be considered for publication.

\section{Manuscript Submission}

General information: Two copies of the manuscript with a letter on the author's letterhead should be submitted to Jack M. Gorman, Editor (or, in Europe, to Joseph Zohar, International Editor), c/o MedWorks Media, 333 Hudson Street, 7th Floor, New York, NY 10013; (F) 212.328.0600. Authors are also required to submit their manuscripts on computer disk in Microsoft Word format. Disks should be labeled with the word processing program, title of paper, and lead author's name. Accepted manuscripts and letters will be edited for clarity and style.

Letters of permission to reproduce previously published material: All material reproduced from previously published copyrighted material must be accompanied by a letter of permission from the copyright holder. All such material should include a full credit line (eg, in the figure or table legend) acknowledging the original source. Any citation of unpublished material or personal communication should also be accompanied by a letter of permission for anyone who is not an author of the paper.
Peer review: Authors must provide five names of parti-cularly qualified potential reviewers with no conflict of interest in reviewing the work. Contact information, including complete address, phone, fax numbers, E-mail address, and affiliations, should be included. The corresponding author will be notified by the editors when a decision regarding acceptance has been made. Peer review is anonymous.

\section{Manuscript Preparation}

Length: Reviews and Original Reports should not exceed 5,000 words (excluding References). Letters should not exceed 1,500 words. Single Case Reports should not exceed 3,750 words and may be submitted with a photograph, if applicable. Diagnostic/treatment algorithms (see Reviews) should contain an extensive introduction, flowchart or series of graphs that fill 8-12 journal pages, and a concise summary.

Spacing: One space should be left after commas and periods. Manuscripts should be double-spaced.

Abstract: Authors must provide a brief abstract.

References: American Medical Association style. See the following examples:

1. Jones J. Necrotizing Candida esophagitis. JAMA. 1980;244:2190-2191.

2. Stryer L. Biochemistry. 2nd ed. San Francisco, Calif: WH Freeman Co; 1980:559-596.

Continuing Medical Education: Authors must submit four multiple-choice questions (two Type $\mathrm{A}$ and two Type $\mathrm{K}$ ), with answers.

Copyright: Materials are accepted for exclusive publication in CNS Spectrums and become the property of CNS Spectrums. Permission to reproduce material must be obtained from the publisher.

\section{Disclosure of Commercial Interests}

Authors must include a statement about all forms of support, including grant and drug company support. Such information may, at the editor's discretion, be shared with reviewers. If the article is accepted for publication, the editors will consult with the authors as to whether this information should be included in the published paper.

\section{Submission Checklist}

Original manuscript plus one copy, with cover letter on author's letterhead

$\square$ Copies of permission letters to reproduce previously published and unpublished material

$\square$ A brief abstract of the article

$\square$ Two CME multiple-choice questions with answers

$\square$ Disk labeled with the word processing program, title of paper, and lead author's name

$\square$ Names and addresses of five potential reviewers 
BRIEF SUMMAAYY Of PRESCRIBING INFORMATIOH
INDICATIOHS ANO USAGE

SEROQUEL is indicated for the treatment of schizophrenia.

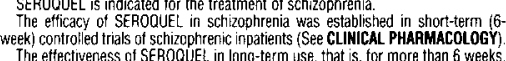

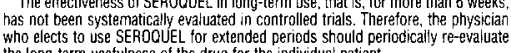
the long-term usetulness
CONTRAIMDICATIOHS

medication or any of its ingredients.

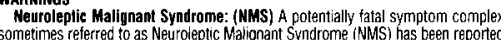
in association with adminisistration of antipsychotic drugs. Two possibls cases of
NMS [2/2387 $(0.1 \%)]$ have been reported in clinical trials with SEROQUEL. Clinical manifestations of NMS a are hyperpyrexia, muscle rigidity, altered mental status, and
evidence of autonomic instability (ivregular pustse or bood pressure, tachycardia, diaphoresis, and cardiaz dysthythmia). Addititonal signn may include elevated creatine rostic evaluation of patients with this syndrome is complicated. in arriving at a
ciagnosis, it is imponant to exclude cases where the clinical presentation includes diagnosis, it is impontant to exclude cases where the clinical presentation includes
both serious medica illness (i.g.g., pneumoniai, systemic infection, etc. jand untreated
of inadequately treated extrapyramidal signs and symptoms (EPS). Other important considerations in the differential diagnosis include centital anticholinergic toxicity,
heat stroke, drug fever and primary central nervous system (CNS) pathology. The management of NMS should include: 1) immediate discontinuation of antipsychotic
drugs and other drugs not essential to concurrent therapy. 2) intensive symmptomatic medical probterms for which specific treatments are available. There is no general

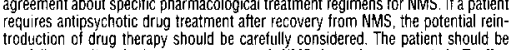

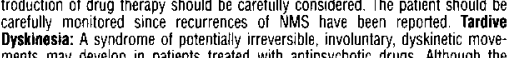
Dyskininesta: A syndrome of potentially irreversible, involuntary, dyskinetic move
ments may develop in patients treated with antipsychotic drugs. Although the

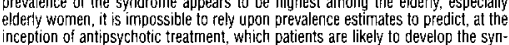
inception of antipsychotic treatment, which patients are likely to develop the syn-
drome Whether antipsychotic drug products difter in their potential to cause tardive that it will become irreversible are believed to increase as the duration of treatment increase. However, the syndrome can devetop, although much less commoniy, after
relatively brief treatrment periods at low doses. There is no known treatment for or completely if articsychotic treatment is withdrawn. Antipsychotic treatment. iselt, however, may suppress for partially suppress) the signs and symptoms of the
syndrome and therebyy may possibly mask the underlying process. The etfect that symptomatic surpression has upon the long-term course of the syndrome is
unknown Given these considerations, SERoautel should be prescribed in a manner that is most tikely to minimize the occurrence of ardive dyskinesia. Chronic antipsy-
chotic treatment should generalily be reserved for patien:ts who appear to suffer from

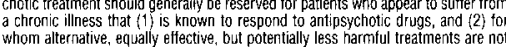
avalable or appropriate. In patients who do require chronic treatment, the smallest
dose and the shortest duration of treatment producing a satisfactory clinical periodically. It signs and symptoms of tardive dyskinesia appear in a patient on

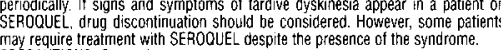
MRE require treatiment w

Orhostatic Hypotension: SEROQUEL may induce orthostatic hypctension associated with dizziness, tachycardia and, in some patients, syncope, especially durino

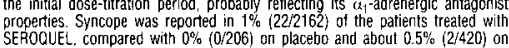
active control drugs. The risk of orthostatic hypotension and syncope may be
minimized by limiting the initial dose to 25 mig bid. it hypotension occurs during Ititration to the target dose, a return to the previous dose in the titration schedule
is appropriate. SEROQUEL shoudid be used with particular caution in patients with known cardioyascular disease (history of myocardial infarction or ischemic heart
dissease. heart failure or conduction abnorma ities). cerebrovascular disease or conditions which would predispose patients to hypotennsion (dachyd ation, hypovolemia and treatment with antithypertensive medicatcons). Calaracts. The development of
cataracts was onserven in association with quefiapine ireatment in chronic dog

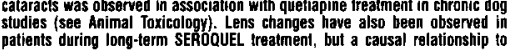
patients during Iong-term SEFOQUEL L Ireatment, but a causal relationship to
SEROOUUEL use has not been established. Nevertheless, the possibility of the lens by methods adequate to detect cataract formation, such as slit lamp exam or other appropriately sensitive methods, is recomommended at initiation of
treatment or shortly thereatier, and at 6 month intervals during chronic treatment. Seizures: During clinical trials, seizures occurred in $0.8 \%$ (18/2387) of patients

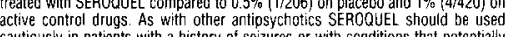
cautiously in patients with a history of seizures or with conditions that potentially
lower the seizure threshold, e.g. Altheimer's dementila. Conditions that lower the Seizure threshold may be more prevalent in a population of 65 years or older.
Hypothyroidism: Clinical trials with SSROQULE demnonstrated a dose-related tecrease in total and free thyroxine (T4) of approximately $20 \%$ at the higher end of
the therapeutic dose range and was maximal in the first two to four weeks of treatment and maintained without adaptation or progression during more chronic therapy
Generally, these changes were of no clinical siggificance and TSH was unchanged
in most patients, and levels ot TBG were unchanged. In nearly all cases, cessation in most patients, and levels of TRG were unchanged. In nearly all cases, cessation
of SEROQUEL treatment was associated with a reversal of the efffects on total and
iree $T 4$. irrespective of the duration of treatment. About $0.4 \%$ (10/23836) of

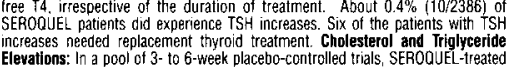
increases needed replacement thyroid treatment. Cholesterol and Triglyceride
Elevations: In a pool of 3 - to 6 -week placebo-controlled trials SEROLutul-treated patients had increases from baseline in cholestercol and triglyceride of $11 \%$ and
$17 \%$, respectively, compared to slight decreases for placebo patients. These
changes were only wegkily related to the increases in weight observed in SEROQUUELtreated patients. Hyperprolactinemia: Altheugh an elevation of prodactir levels was

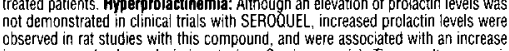
in mammary gland neoplasia in rats (see Carcinogenesis). Tissue culture experi-
ments sindicate that approximately one--hirrot houman breast cancers ares prolactin dependent in vitro, a tactor of potential importance if the prescription of these drugs
is contemplated in a patient with previously detected breast cancer. Although

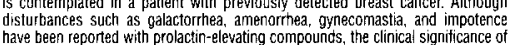
elevated serum prolactin levels is unknown for most patients. Neither clinical studies nor epidemiologic studies conducted to date have shown an association between avalabie evidence is considered to limited to be corclusive at this time.
Transaminase Elevations: Asymptomatic, transient and reversible elevations in

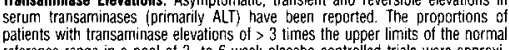
efererence range in a pool of 3-to 6-week placebo-controlled trials were approxielevations usually occurred within the first 3 weeks of drug treatment and promptly
returned to pre-study levels with ongoing treatment with SEROQULE. Potential tor Cognitlve and Motor Impairment: Somnotence was a commonly yeported adverse
event reponted in patients treated with SEROOUEL especially durning the $3-5$ day

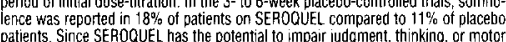
skills, palients should be cautioned about performing activities requiring mental alertness, such as operating a motor vehicle (including automobiles) or operating
hazardous machinery untif they are reasonably certain that SEROCOUEL therapy SEROOUEL has been reported prior to miarket introduction. While a causal relatitionship to use of SEROQUEL has not been established, other drugs with
alpha-adrenergic blocking effects have been reported to induce priapism, and it is

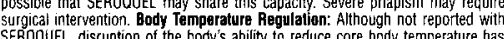
SEROOUEL, disruption of the body's ability to reduce core body temperature has
been attributed to antipsychotic agents. Appropriate care is advised when prescribibg
SEROQUEL for patients who will be experiencing conditions which may contribute to an elevation in core body temperatsree, e.g. exeectising strenuously exposure to
extreme heat, receiving concomitant medication with anticholinergic activity, or
SEROQUEL ${ }^{(9)}$ (quetiapine fumarate) Tablets

have been associated with antipsychotic drug use. Aspiration pneurnonia is a commen cause of morbidity and mortality in elderly patients, in particulats those wit should be used cautiously in patients at risk for aspiration pneumonia. Suicied The possibility of a suicide attempt is inherent in schizophrenia and close SUpervision
of high risk patients should accompany drug therapy. Prescriptions tor SEROOUEL
should be written for the smallest management in order to reduce the risk of overdose. Use in Patients with Concomitant Illness: Clinical experience with SEROQUEL in patients with certain used to any appreciable extent in patients with a recent history of myocardial infarcpremarketing clinical studies. Becauss of the risk of orthostatic hypotension with SEROQUEL, caution should be observed in cardiac patients (see OOrthostatiti
Hypotension) Intormation for Patients: Physicians are advised to discuss the Hypotension). Intormation for Patients: Physicians are advised to discuss the
following issues with patients for whom they prescribe SEROQUEL. Orthostatic
Hypotension: Patients should be advised of the risk of orthostatic hypotension especially during the 3-5 day period of initial dose titration, and also at times re-initiating treatment or increases in dose. Interference with Cognitive and Mol
Pertormance: Since somnolence was a commonly reported adverse event associater Wwith SEROQUEE treatment, patients should be advised of the risk of Somnclence
especially during the 3-5 cay period of initial dose titration. Patients shoukd be

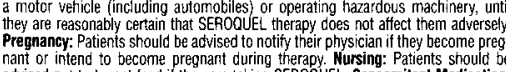

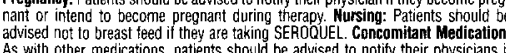
they are taking, or plan to take, any prescription or over-the-counter drugs. Alcohol: Patients should be advised to avoid consuming alcoholic beverages while taking
SERODUUEL. Heal Exposure and Dehlydration: Patients shoutd be advised regardin appropriate care in avviding overtheating and dehydration. Laboratory Tessts: $N$ $S E R O O U E L$
systematic studies. Given the primary CNS effects of SEROQUELL, caution should b used when it is taken in combination with other centrally acting, drugs. SEROOUE
potentiated the cognitive and motor eftects of alcohol in a clinical trial in subjects with selected DSychotic disorders, and aicoholic beverages should be avoided while
taking SEROQUUf. Because of its potential tor inducing hypotension. SEROOLE

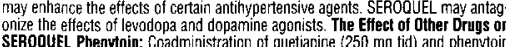

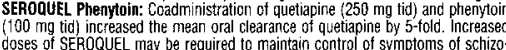
phrenia in patients recelving quetiapine and phenytoin, or other hepatic enzzym

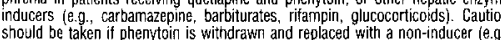
valproate). Thioridazine: Thioridazine (200 mg bid) increased the oral clearance quetiapine (300 mg bid) by $65 \%$. Cimetiditine: Administration of multiple daily
coses of cimetidine (400 mo tid tor 4 days) resultited in a $20 \%$ decrease in the mean oral clearance of quetiapine $(150 \mathrm{mg}$ tid). Dosage adjustment for quetiapine is not
required when it is given with cimetidine. $\mathrm{P450} 3 \mathrm{~A}$ Inhibitiors: Coadministration xetoconazale $(200 \mathrm{mg}$ once daily for 4 days , a potent innibitor of cytochrome P450 maximum plasma concicentration of quetiapine. Caution is indicated when SEROOUIE

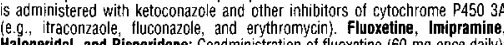
Haloperidol, and Risperidone: Caadministration of fluoxetine $(60 \mathrm{mg}$ once daily) quetiapine $300 \mathrm{mg}$ bidy did not alter the steady-state pharmacokinetics of of lorazepan ( $2 \mathrm{mc}$, single dosel was reduced by $20 \%$ in the presence of quetapin
administered as $250 \mathrm{mg}$ tid dosing. Lithium: Concomitant adminisistration of

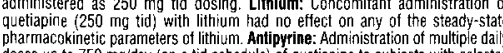
doses up to $750 \mathrm{mg}$ /day (on a tid schedule) of quetiapine to subjects with selected
psychotic disorders had ho cinically yelevant effect on the elearance of antipyrine 0 does not significaritly induce hepatic encymes responsible for cytachrome $P 450$ Fertility Carcinogenesis: Carcinogenicity studies were conducted in C57BL mic
and Wistar rats. Quetiapine was administered in the diet to mice at doses of 20.75 ,

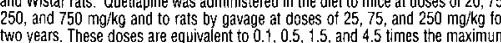

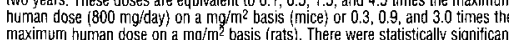
maximum humban dose on a $\mathrm{mg}^{2} \mathrm{~m}^{2}$ basis (rats). There were statistically significicat
increases in thyroid gland follicular adenomas in male mice at doses of 250 and
$750 \mathrm{mg} / \mathrm{kg}$ or 1.5 and 4.5 times the maximum human dose on a

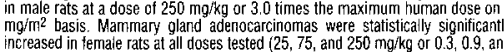
3.0 timas the maximum recommended human dose on a sing $\mathrm{m}^{2}$ basisy. Thyroi
follicular cell adenomas may have resulted trom chronic stimulation of the thyroid gland by thyroid stimulating hormone (TSH) resulting from enhanced metabolis and clearance of thyroxine by rodent liver. Changes in TSH, thyroxine, and thyroxin
clearance consistent with this mechanism were observed in subchronit toxicity
studies in rat and mouse and in a 1 -year toxicity study in rat: however, the results

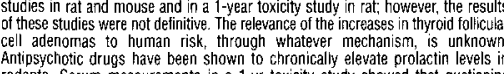
rodents. Serum mansurements 1 in a 1 -yr toxicicty study showed that quetiapin
increased median serum prolactin levels a maximum of 32 - and 13 -fold in male and female rats, respectively. Increases in mammary neoplasms have been found
rodents atter chronic administration of other antipsychotic drugs and are considered mediated mammary gland tumors in rats to human risk is unknown (se Hyperprolactinemiai in PREECAUTIONS, General). Mutagenesis: The mutagenic
potential of quetiapine was tested in six in vitro bacterial gene mutation assays and in an in vitto mammal lian gene mutation assay in Chinese Hamster Ovary cells.
However, sufficiently high concentrations of quetiapine may not have been used tor all tester strains. Ouetiapine did produce a reproducible increase in muttations in on Salmonelia tyobimurium tester strain in the presence of metablicic activation. No assay in cultured human lymphocytes or in the in vivo micronucleus assay in rat.

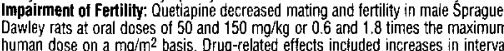
to mate and in the number of matings required for successful impregnation, These effects continued to be observed at $150 \mathrm{mg} / \mathrm{kg}$ even after a two-week period withou treatment. The no-effect dose for impaired mating and fertility in male rats was
$25 \mathrm{mg} / \mathrm{kg}$, or 0.3 times the maximum human dose on a $\mathrm{mg}^{\prime} \mathrm{m}^{2}$ basis. Quetiapin

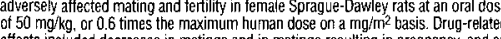
effects included decreases in matings and in matings resulting in pregnnancy, and a
increase in the interval to mate. An increase in irregular estrus cycles was observer at doses of 10 and $50 \mathrm{mgg} / \mathrm{kg}$, or 0.1 and 0.6 times the maximum human dose on
$\mathrm{mg}^{2} \mathrm{~m}^{2}$ basis. The no-effect dose in female rats was $1 \mathrm{mg} / \mathrm{kg}$, or 0.01 times the

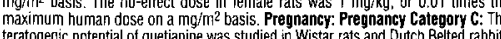
teratogenic potential of quetiapine was studied in Wistar rats and Dinch Betted rabbits
dosed during the period of organogenesis. No evidence of a teratogenic effect was
detected in rats at doses of 25 to 200 mog $/ \mathrm{kg}$ or 0.3 to 2.4 times the maximum detected in rats at doses of 25 to $200 \mathrm{mg} / \mathrm{kg}$ or 0.3 to 2.4 times the maximu
human dose on a mo/m² basis or in rabbits at 25 to $100 \mathrm{mg} / \mathrm{kg}$ or 0.6 to 2.4 times the

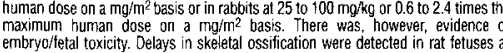

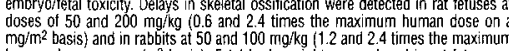

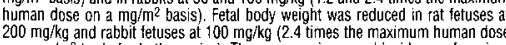
on a magrtin basis for both speciest. There was an increased incidence of a mino (2.4 times the maximum human dose on a mo/m² basis). Evidence of maternal tor-
icity (i.e. decreases in brdy weight gain and/or death) was observed at the highcose in the rat study and at all doses in the rabbit study. In a peritpostnatal repro-
ductive study in rats, no drug-related effects were observed at doses of 1,10 , and

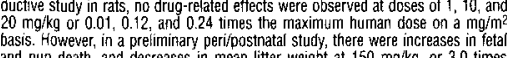

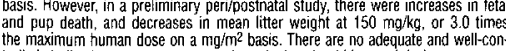
trolled stutuies in pregnant women and puetiapine should be used during pregnancy
onlly if the potential benefit justifites the potential risk to the fetus. Labor and
Delivery: The effect of SEROQUUEL on labor and delivery in humans is unknown.
SEROQUEL (quetiapine fumarate) Tablets

Nursing Mothers: SEROQUEL was excreted in milk of treated arimals during lacta-

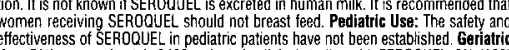
Use: Of the approximately 2400 patients in clinical studies with SEROQUEL, $8 \%$; 190 ) tolerability of SERODOUEL in the elderly compared to younger adults. Nevertheless, th macodynamic response to SEROQUEL, or cause pcorer toterance or orthostasis
chould lead to consideration of a lower starting dose, slower titration. and careffy monitoring during the initial dosing period in the elderly The mean plasma clearance
of SEROOUEL was reduced by $30 \%$ to $50 \%$ in elderly patients when compared to Younger patients.

Adverse Events Occurring at an Incidence of $1 \%$ or More Among SERoqueL Treated Patients in Stort-Term. Placebo-Controlled Trials: The most corninnonly greater) and observed at a rate on SEROQUEL at least twice that of placebo wer The following treetment-emergent adverse experiences occurred at an incidence rate
of $1 \%$ or mare, and were at lieast as frequent among SEROOLIEL treated patients, of $1 \%$ or more, and were at least as frequent among SEROCLiEL treated patients,
treated at doses of $75 \mathrm{mg} /$ day or or greater than amorg placebo treated patients in Body as a Whole: Headache, Asthenia, Abdominal pain, Back pain, Fever; Nervous Dyspepsia: Cardiovascular System: Postural hypotension, Fachycardia; Metabolic
and Nultritional Disorders: Weight gain, Skin and Appendages: Rash: Respiratory

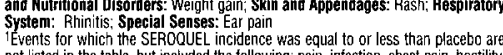
Events for which the SEROQUEL incidence was equal to or less than placebo a a
not isted in the table, but inciuded the tollowing: pain, infection, chest pain, hostility

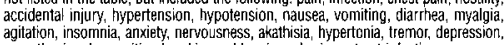
paresthesia, pharyngititis, dry skin, amblyopia ardi urinary tract intection.
Explorations for interactions on the basis of gender, age, and race did not reveat any

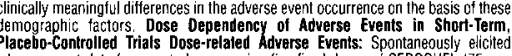
dverse event data from a study comparing five fixed doses of SERODLUEL $775 \mathrm{mg}$. $50 \mathrm{mg}, 300 \mathrm{mg}, 600 \mathrm{mg}$, and $750 \mathrm{mg} /$ dayl to placebo were explored for dose-
eelatedness of adverse events. Logistic regression analyses revealed a positive dos

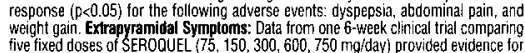
five fixed doses of $S E R O Q U E L$
the lack of treatment-emergent extrapyramidal symptoms (EPS) and dose-relatedness
to $E P S$ S EPS: (1) Simpson-Angus total score (mean change from baseline) which evaluates (akathisia, akinesia, cogwheel rigidity, extrapyramidal syndrome, hypertonia, hypoki-
nesia, neck rigidity, and tremor). and (3) use of anticholinergicic medications to treat emergent EPS. In three additional placebo-control ed clinical trials using variable
doses of SEROQUEL, there were no differences between the SEROOLUEL and placebo

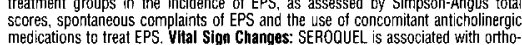

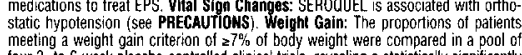
four 3 - to 6 -week placebo-controlled clinicat trais, revealing a statistically significantly

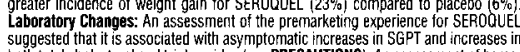

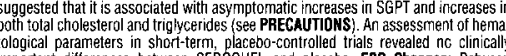
tological parameters in short-term, placebo-controlled trials revealed nc clinically
mportant differences between SEROAUEL and placebo. ECG Changes: Between group comparisons for poeled plocebo-controiled trials reveazlad no statistically

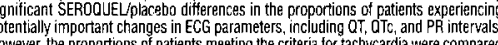
four 3- 10 6-week placebo-controlled clinical trials revealing a $1 \%$ (4/399) incidenc

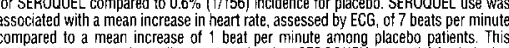

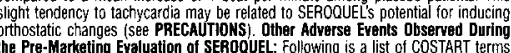
that reflect treatment-emergent adverse events as defined in the introduction to the ADVERSE REACTIONS section reportred by patients treated with SEROOUUEL at multiple doses z 75 mgrday during any phase of a trial within the premarketing database of
approximately 2200 patients. All reported events are inciuded except thase already a
remote, and those event terms which were so general as to be uninformative. It is important to emphasize that, although the events reported occurred during treatmen by body system, and listed in order of decreasing frequency according to the following anty those not already yisted in the tabulated results srom placebo-controlled trials
opear in this listing); initrequent adverse events are those occurring in $1 / 100$ to appear in this listing); intrequent adverse events are those occurring in 1/1000 to
$1 / 1000$ patients; rare events are those occurring in tewer than 11000 patients.
Nervous system: Frequent: hypertonia, dysarthria; infrequent: abnormal dreams. dyskinesia thinking abnormal, tardive dyskinesia, vertigo, involuntary movements,
contusion, amnesia, psychosis, hallucinations, hyperkinesia, libido increased ${ }^{*}$, urinary retention, incoordination, paranoid reaction, abnormal gaat, myoclonus, delusions, manic reaction, apathy, ataxia, depersonalization, stupor, bruxism, catatonic reaction
hem iplegia; Rare: aphasia, buccoglossal syndrome, choreagathetosis, delirium, emo-
tional labilithy, euphoria, libido decreased ${ }^{*}$, neuralgalgia, stuttering, subdural hematoma tional lability, euphoria, ibido decreased ${ }^{*}$, neuralgia, stuttering, subdural hematoma attempt, malaise, photosensitivity reaction, chills, tace edema, moniliasis; Rare
abdomen enlarged. Digestive System: Frequent: anorexia; infrequent: increased salivation, increased appetite, gamma glutamyl transpeptidase increased, gingivitis,
ddysphanic flatulence gastroenteritis gastritis, hemorrnoids, stomatitis, thirst tooth caries, fecal incontinence, gastroesoophageal reflux, qum hemorrhage, mouth ulceration, rectat hemornage, tongue edema; Rare: glossitis, hematemesis, intestinal obstruction,
melena, pancreatitis . Cardiovasuluar system: Frequent: palpitation; Intrequent. vasodilitation, OT interval prolonged, migraine, bradycardia, cerebral ischemia,
irregular pulse, I wave abnormality, bundle branch block, cerebrovascular accident,
deep thrombophlebitis, T wave inversion, Bare: anginiz: pectoris, atrial fibrillation. AV block first degree, congestive heart failure, ST elevated, thrombophlebitis, T waye flat-
tening, ST abnormality, inceased QRS duration. Respiratory Systemm: Frequent: pharyngitis, rhinitis, cough increased, dyspnea; imfrequent: pneumonia, epistaxis,
asthma: Rare: hiccup, hyperventilation. Metabolic and Nutritional Systemn: Frequent: peripheral edema; infrequent: weight loss, alkaline phosphatase increased
hyperlipemia, alcohol intolerance, dehydration, hypergycemia, creatinine increased hypoglycemia', Rare: glycosuria, goutt, hand edema, hypokalemia, water intoxication

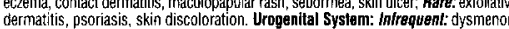

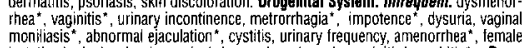

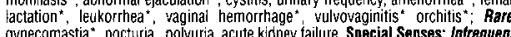
conjunctivitis, abnormal vision, dry eyes, tinnitus, taste perversion, blepharitis, eye
pain; Rare: abnormality of accommodation, deatness, glaucoma. Musculoskeletata System: Imfrequent: pathological fracture, myasthenia, twitching. arthralgia, arthritis. Infrequent: leukocytosis, anemia, ecchymosis, eosinophilia, hypochromic anemia Imfrequent: : which were temporally related to SEROQDUELL therapy include the forllowing: rarel sible risk factors for leukopenianneutropenia incluc-

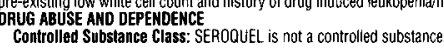




\section{FHBTF-UINE TREATMEHT FOR SGHIZOPHRENIA}

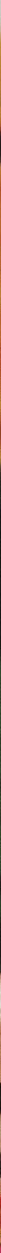

\section{WEII!}

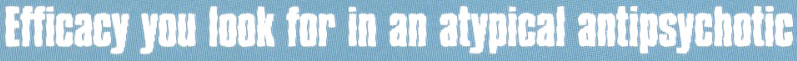

- Proven to reduce positive and negative symptoms'

\section{AREFPTED!}

An argellent side-efiest profile

- The only first-line atypical antipsychotic with an EPS*

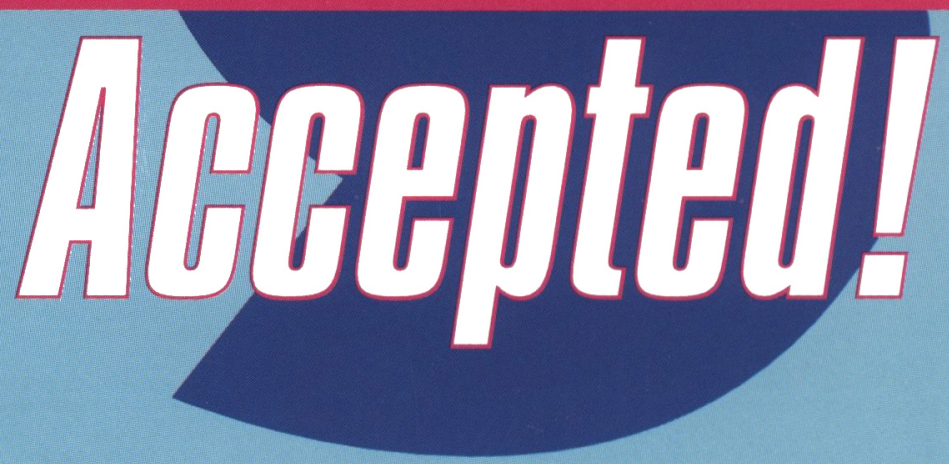
profile no different from placebo across the entire dosing range (up to $800 \mathrm{mg}$ ).

*Extrapyramidal symptoms.

- The most common adverse events associated with the use of SEROQUEL are dizziness $(10 \%)$, postural hypotension $(7 \%)$, dry mouth $(7 \%)$, and dyspepsia $(6 \%)$. The majority of adverse events are mild or moderate

- As with all antipsychotic medications, prescribing should be consistent with the need to minimize the risk of tardive dyskinesia, seizures, and orthostatic hypotension

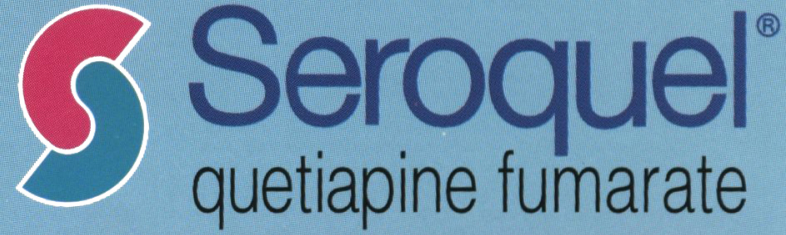

$25 \mathrm{mg}, 100 \mathrm{mg}, 200 \mathrm{mg} \& 300 \mathrm{mg}$ tablets

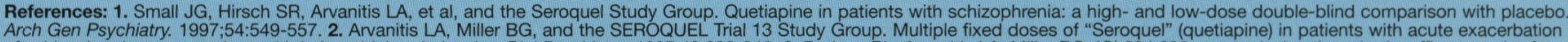

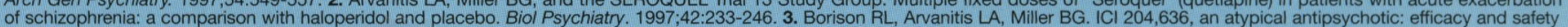
in a multicenter, placebo-controlled trial in patients with schizophrenia. J Clin Psychopharmacol, 1996;16:158-169. 4. Data on file, Study S91, AstraZeneca Pharmaceuticals LP. Wilmington, Delaware. 5. SEROQUEL ${ }^{\circ}$ (quetiapine fumarate) Prescribing Information, Rev 1/01, AstraZeneca Pharmaceuticals LP. Wilmington, Delaware.

\section{AstraZeneca}

